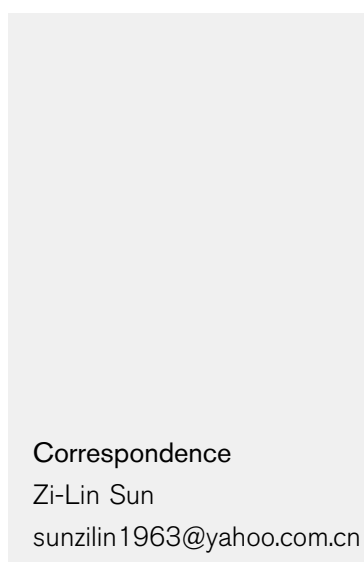

Received 14 March 2010

Accepted 28 June 2010

\section{Meticillin-resistant Staphylococcus aureus isolated from foot ulcers in diabetic patients in a Chinese care hospital: risk factors for infection and prevalence}

\author{
Shao-Hua Wang, ${ }^{1}$ Zi-Lin Sun, ${ }^{1}$ Yi-Jing Guo, ${ }^{2}$ Bing-Quan Yang, ${ }^{1}$ \\ Yang Yuan, ${ }^{1}$ Oiong $\mathrm{Wei}^{1}$ and Kuan-Ping $\mathrm{Ye}^{1}$ \\ ${ }^{1}$ Department of Endocrinology, Affiliated Zhongda Hospital of Southeast University, Nanjing \\ 210009, PR China \\ ${ }^{2}$ Department of Neurology and Institution of Cerebral Vascular Disease, Affiliated Zhongda Hospital \\ of Southeast University, Nanjing 210009, PR China
}

\begin{abstract}
A retrospective case-control study of 118 (male : female, 68:50) Chinese type 2 diabetic patients with foot ulcers (Wagner's grade 3-5) was conducted to determine the prevalence and risk factors for meticillin-resistant Staphylococcus aureus (MRSA) infection in relation to the original community or hospital parameters. Ulcer specimens were processed for Gram staining, aerobic culture and antimicrobial susceptibility testing. Staphylococcus species were tested for meticillin resistance using oxacillin. $S$. aureus was the most frequent pathogen $(25.6 \%)$ in diabetic patient specimens (160 isolates), and a high proportion of $S$. aureus isolates were MRSA $(63.4 \%)$. A high percentage of $S$. aureus isolates $(65.4 \%)$ satisfied the definition for hospitalassociated MRSA (HA-MRSA) infection. The size of ulcers [adjusted odds ratio (OR) 1.61; $95 \%$ confidence interval (Cl) 1.22-2.12] and osteomyelitis (adjusted OR 18.51, $95 \% \mathrm{Cl} 2.50-$ 137.21) were independent predictors of MRSA infection. The HA-MRSA group had a significantly different distribution from the community-associated MRSA group with respect to age, history of diabetes and length of hospital stay (all $P<0.001$ ). Neuropathy, vascular disease (all $P=0.049$ ) and osteomyelitis $(P=0.026)$ were the most common underlying conditions observed in the HAMRSA group. This study contributes to the establishment of precautions against the emergence of MRSA including MRSA acquired from different sources among the Chinese population with diabetic foot ulcers based on their original or clinical parameters.
\end{abstract}

\section{INTRODUCTION}

Foot lesions in diabetic patients are a major medical, social and economic problem. Infectious agents are associated with amputation of the infected foot if not treated promptly. Infection with multidrug-resistant organisms may increase the duration of hospital stay and cost of management, as well as morbidity and mortality (Hartemann-Heurtier et al., 2004).

Staphylococcus aureus is the most common pathogen among Gram-positive bacteria isolated from ulcers. Almost $50 \%$ of $S$. aureus isolates are meticillin-resistant $S$. aureus (MRSA). MRSA has been increasingly isolated from diabetic foot ulcers, and several studies have found its emergence in as

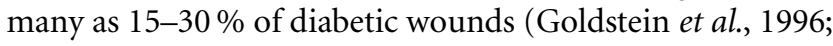

Abbreviations: $\mathrm{CA}$, community-acquired; $\mathrm{Cl}$, confidence interval; $\mathrm{HA}$, hospital-associated; $\mathrm{HbA}_{1 \mathrm{c}}$, glycosylated haemoglobin; MRSA, meticillinresistant Staphylococcus aureus; OR, odds ratio.
Shankar et al., 2005; Tentolouris et al., 1999). MRSA emergence may be associated with adverse prognosis. Patients with community-acquired MRSA (CA-MRSA) infections often do not exhibit the risk factors known in patients with hospital-associated MRSA (HA-MRSA) infections. These risk factors include recent hospitalization, dialysis, nursing-home residence and the presence of other co-morbid conditions such as diabetes, chronic renal failure and chronic pulmonary diseases, which bring patients into contact with healthcare settings.

However, the clinical characteristics and factors that predispose diabetic patients with foot ulcers towards infection with different associated MRSA remain unclear. This study aimed to compare the prevalence and antimicrobial susceptibility profile of CA-MRSA with HAMRSA isolated from Chinese diabetic patients with foot ulcers. The risk factors for infection of ulcers with MRSA and the outcome of these infections were also studied. 


\section{METHODS}

Patients and infection definition. Samples for this retrospective study were collected from 118 type 2 diabetic patients with infected foot ulcers (Wagner's grade 3-5). The patients were admitted to the Diabetic Foot Care Center at the Zhongda Hospital of Southeast University, Nanjing, PR China, from 1 January 2004 to 31 December 2006. Infection was diagnosed according to the criteria proposed by the International Consensus on the Diabetic Foot (Lipsky et al., 2004). Ulcers were graded using the Wagner classification (Wagner, 1981). The control group comprised 97 type 2 diabetic patients with foot ulcers (Wagner's grade 3-5) without CA-MRSA or HA-MRSA infection.

HA-MRSA infection is defined as that occurring in a patient whose MRSA isolate was cultured more than $48 \mathrm{~h}$ after admission, who has a history of hospitalization of more than $24 \mathrm{~h}$, who has had dialysis, surgery or residence in a long-term healthcare facility within 6 months prior to the culture date, or who has an indwelling intravenous line, catheter or any other percutaneous medical device present at the time the culture was taken. CA-MRSA is defined as an MRSA isolate obtained within $48 \mathrm{~h}$ of hospitalization from a patient without the above-mentioned risk factors (Fridkin et al., 2005; Naimi et al., 2003). Patients who had infections in other body sites or an MRSA-positive isolate prior to the study period were excluded from the study.

Data collection. Data were collected via a case record form. The data included demographic data, duration of diabetes, glycaemic control and presence of retinopathy, nephropathy (creatinine $\geqslant 150 \mathrm{~mol} \mathrm{l}^{-1}$ or the presence of micro- or macroalbuminuria), neuropathy (absence of perception of the Semmes-Weinstein monofilament at two of ten standardized plantar sites on either foot) and peripheral vascular disease (ankle brachial pressure index $<0.9$, or a history of intermittent claudication or rest pain, with or without pedal pulses).

The duration and size of the ulcer, clinical outcome, duration of hospital stay and glycaemic control during the hospital stay were noted for each patient. Clinical assessment for signs of infection (swelling, exudate, surrounding cellulitis, odour, tissue necrosis, crepitation and pyrexia) was performed. Hospitalizations within the previous 6 months and residence in nursing homes were systematically recorded. Laboratory values were determined at the laboratory centre of Zhongda Hospital.

Ulcer size, expressed in $\mathrm{cm}^{2}$, was determined by multiplying the longest and widest diameters. Osteomyelitis was diagnosed from suggestive changes in radiographs and bone scans. All patients were monitored until discharge from the hospital.

Microbiological methods. Each ulcer specimen for culture was obtained at least twice, once within $48 \mathrm{~h}$ and the other more than $48 \mathrm{~h}$ after admission, both taken after the surface of the wound had been washed vigorously with saline, followed by debridement of superficial exudates. Specimens were obtained by scraping the ulcer base or the deep portion of the wound edge with a sterile curette, and were promptly sent to the laboratory to be processed for Gram staining, aerobic culture and antimicrobial susceptibility testing.

Characterization of isolates. All MRSA cultures were confirmed in the microbiology laboratory of Zhongda Hospital. Antimicrobial susceptibility testing of aerobic isolates was performed using a standard disc diffusion method according to the recommendations of the National Committee for Clinical Laboratory Standards (NCCLS, 2002). Susceptibility to gentamicin, tetracycline, erythromycin, clindamycin, nitrofurantoin, fusidate, sulfamethoxazole, rifampicin, vancomycin, teicoplanin, levofloxacin, quinupristin/dafopristin, penicillin, minocycline and norfloxacin was determined. Staphylococcus species were tested for meticillin resistance using a $1 \mu \mathrm{g}$ oxacillin disc and oxacillin screen agar $\left(6 \mu \mathrm{g}\right.$ oxacillin $\left.\mathrm{ml}^{-1}\right)$.
Statistical analysis. Quantitative data were expressed as means \pm SD, and qualitative variables were expressed as frequencies. For statistical analysis, Fisher's exact test, Pearson's $\chi^{2}$ test and Student's $t$-test were used as appropriate. Continuous variables were analysed using a twotailed $t$-test, and $P<0.05$ was considered statistically significant. For the case-control study, conditional univariate and multivariate logistic regression models were used to estimate the odds ratio (OR) and the associated $95 \%$ confidence interval (CI). All statistical analyses were performed using statistical software (SPSS for Windows, version 11.5).

\section{RESULTS}

\section{Characteristics of patients and wounds}

Over half of the patients in the study were male (57.6\%). The mean age of the subjects was $60.8 \pm 10.4$ years. The mean duration of diabetes was $8.8 \pm 7.0$ years, and $46(39.0 \%)$ had had the condition for $\geqslant 10$ years. The mean level of glycosylated haemoglobin $\left(\mathrm{HbA}_{1 \mathrm{c}}\right)$ was $7.4 \pm 1.9 \%$; 87 patients $(73.7 \%)$ had neuropathy, $88(74.6 \%)$ had peripheral vascular disease, 59 (50.0\%) had nephropathy, 48 $(40.7 \%)$ had retinopathy and $80(67.8 \%)$ were hypertensive. Osteomyelitis was present in 20 patients (16.9\%).

\section{Total number of bacteria and specific strains}

A total of 160 isolates were detected from the 118 ulcer specimens, giving a mean of 1.4 species per patient. Nearly $40 \%$ of patients had an infection from two or three species, whilst infection from three species was found in $6.0 \%$ of patients.

A profile of the organisms isolated is given in Table 1. Of the 160 isolates, $81(50.6 \%)$ were Gram-positive aerobic bacteria and eight were fungal isolates $(5.0 \%)$. Of the 81 Gram-positive bacterial isolates, the majority were Staphylococcus species $(n=51 ; 63.0 \%)$. S. aureus was the

Table 1. Profile of bacteria isolated from infected foot ulcers in specimens from diabetic patients ( $n=118$ patients)

The total number of isolates was 160 .

\begin{tabular}{|lc|}
\hline Category & Frequency (\%) \\
\hline Gram-negative bacteria & \\
Proteus species & $17(10.6)$ \\
Escherichia coli & $32(20.0)$ \\
Pseudomonas aeruginosa & $11(6.9)$ \\
Acinetobacter species & $7(4.4)$ \\
Klebsiella species & $4(2.5)$ \\
Gram-positive bacteria & \\
Staphylococci species & 51 \\
S. aureus & $41(25.6)$ \\
S. haemolyticus & $4(2.5)$ \\
S. epidermidis & $6(3.8)$ \\
Enterococcus species & $17(10.6)$ \\
Streptococci & $13(8.1)$ \\
Fungi & $8(5.0)$ \\
\hline
\end{tabular}


most frequent pathogen, found in about one-quarter of the total infections $(41 ; 25.6 \%)$. In our data, a high proportion $(n=26 ; 63.4 \%)$ of $S$. aureus isolates were MRSA; 17 $(65.4 \%)$ satisfied the definition of HA-MRSA, whilst the rest $(n=9 ; 34.6 \%)$ were classified as CA-MRSA.

\section{Characteristics of patients infected with MRSA and their wounds}

Twenty-one type 2 diabetic patients (Wagner's grade 3-5) with foot ulcers were infected with MRSA. The clinical features of the cases and controls are given in Table 2. They did not differ significantly in gender, duration of ulcer or duration of hospital stay. Chronic diabetic complications, Wagner grade and discharge status were not associated with MRSA infection. Age, duration of diabetes, level of $\mathrm{HbA}_{1 \mathrm{c}}$, size of ulcer and osteomyelitis were significantly associated with CA-MRSA infection. The number of MRSA-infected patients that had an increased requirement for surgical treatment was also significantly higher than controls.

Six variables were eligible for inclusion in the multivariable model. In this model (Table 3), the size of ulcer (adjusted OR 1.61; $95 \%$ CI 1.22-2.12) and osteomyelitis (adjusted
OR 18.51; $95 \%$ CI 2.50-137.21) were independent predictors of MRSA infection. Age, duration of diabetes, $\mathrm{HbA}_{1 \mathrm{c}}$ and requirement for surgical treatment were not statistically significant predictors of MRSA infection in the multivariable model.

The HA-MRSA group had significantly different distributions from the CA-MRSA group with respect to age and length of hospital stay (both $P<0.001$; Table 2). Patients with HA-MRSA ulcers had a long history of diabetes $(P=0.001)$; neuropathy, vascular disease (both $P=0.049$ ) and osteomyelitis $(P=0.026)$ were the most common underlying conditions observed in the HA-MRSA group. There was no significant difference between CA-MRSA and HA-MRSA in either duration or size of ulcer, although HA-MRSA colonization of ulcers was characterized by longer histories and larger ulcers compared with those of CA-MRSA.

\section{Antimicrobial susceptibility in patients infected with MRSA and their prognosis}

The prevalence of MRSA resistance to each antimicrobial tested is presented in Table 4. CA-MRSA isolates were more likely than HA-MRSA isolates to be susceptible to

Table 2. Association of study characteristics in two groups of diabetic patients according to infection of foot ulcers with HA-MRSA and CA-MRSA

Twenty-one type 2 diabetic patients (Wagner's grade 3-5) with foot ulcers were infected with MRSA. The control group included 97 type 2 diabetic patients with foot ulcers (Wagner's grade 3-5) without CA-MRSA or HA-MRSA infection.

\begin{tabular}{|c|c|c|c|c|c|c|c|}
\hline Characteristic & MRSA & Control & OR $(95 \% \mathrm{CI})$ & $P$ value & HA-MRSA & CA-MRSA & $P$ value \\
\hline Age (years) & $66.8 \pm 8.1$ & $59.4 \pm 10.5$ & $1.10(1.03-1.17)$ & 0.004 & $71.2 \pm 5.3$ & $58.0 \pm 4.7$ & $<0.001$ \\
\hline Male & 15 & 53 & $2.08(0.74-5.80)$ & 0.164 & 10 & 5 & 1.00 \\
\hline Female & 6 & 44 & & & 4 & 2 & 1.00 \\
\hline Duration of diabetes (years) & $14.1 \pm 8.3$ & $7.6 \pm 6.2$ & $1.13(1.05-1.21)$ & 0.001 & $17.9 \pm 7.4$ & $6.4 \pm 2.9$ & 0.001 \\
\hline Duration of ulcer (months) & $1.5 \pm 1.6$ & $1.0 \pm 1.4$ & $1.22(0.92-1.62)$ & 0.168 & $1.8 \pm 1.9$ & $1.1 \pm 0.5$ & 0.361 \\
\hline Duration of hospital stay (days) & $26.4 \pm 13.7$ & $24.9 \pm 8.7$ & $1.02(0.97-1.06)$ & 0.527 & $33.1 \pm 11.9$ & $13.0 \pm 2.7$ & $<0.001$ \\
\hline Wagner's grade & - & - & $1.77(0.75-4.20)$ & 0.196 & - & - & - \\
\hline Size of ulcer $\left(\mathrm{cm}^{2}\right)$ & $6.5 \pm 3.8$ & $2.5 \pm 1.9$ & $1.69(1.34-2.12)$ & $<0.001$ & $7.1 \pm 4.3$ & $5.3 \pm 2.3$ & 0.334 \\
\hline Case with number of ulcers $\geqslant 2$ & 7 & 10 & $2.83(0.92-8.71)$ & 0.069 & 6 & 1 & 0.19 \\
\hline \multicolumn{8}{|l|}{ Complications } \\
\hline Peripheral vascular disease & 17 & 71 & $1.56(0.48-5.06)$ & 0.462 & 13 & 4 & 0.049 \\
\hline Osteomyelitis & 13 & 7 & $20.89(6.49-67.27)$ & $<0.001$ & 11 & 2 & 0.026 \\
\hline \multicolumn{8}{|l|}{ Treatment } \\
\hline Interventional & 3 & 4 & $3.88(0.80-18.81)$ & 0.093 & 1 & 2 & 0.186 \\
\hline Surgical & 6 & 7 & $5.14(1.52-17.42)$ & 0.009 & 6 & 0 & 0.04 \\
\hline \multicolumn{8}{|l|}{ Discharge status } \\
\hline Alive & 20 & 96 & $0.21(0.01-3.47)$ & 0.274 & 13 & 7 & 0.469 \\
\hline Dead & 1 & 1 & $0.21(0.01-3.47)$ & 0.274 & 1 & 0 & 0.469 \\
\hline
\end{tabular}


Table 3. Multivariate conditional regression analysis of risk factors for MRSA infection: matched case-control study

Twenty-one type 2 diabetic patients (Wagner's grade 3-5) with foot ulcers were infected with MRSA. The control group included 97 type 2 diabetic patients with foot ulcers (Wagner's grade 3-5) without CAMRSA or HA-MRSA infection.

\begin{tabular}{|lcc|}
\hline Characteristic & OR $(\mathbf{9 5} \% \mathbf{C I})$ & $\boldsymbol{P}$ value \\
\hline Age (years) & $1.02(0.94-1.12)$ & 0.606 \\
Duration of diabetes (years) & $1.02(0.92-1.13)$ & 0.735 \\
$\mathrm{HbA}_{1 \mathrm{c}}(\%)$ & $1.15(0.78-1.71)$ & 0.488 \\
Size of ulcer $\left(\mathrm{cm}^{2}\right)$ & $1.61(1.22-2.12)$ & 0.001 \\
Osteomyelitis & $18.51(2.50-137.21)$ & 0.004 \\
Surgical treatment & $0.53(0.06-4.97)$ & 0.527 \\
\hline
\end{tabular}

clindamycin $(P=0.012)$, sulfamethoxazole $(P=0.019)$, rifampicin $(P=0.042)$, levofloxacin $(P=0.031)$ and norfloxacin $(P=0.001)$. Surgical debridement was performed in six MRSA patients, all of whom were HA-MRSA patients.

\section{DISCUSSION}

MRSA has emerged as a serious and commonly occurring problem in diabetic patients with foot ulcers (Game \& Jeffcoate, 2004; Tentolouris et al., 1999). This study presents a comprehensive clinical and microbiological survey of Chinese hospitalized diabetic patients with foot ulcers infected with MRSA. We focused on the prevalence of and factors that predispose patients to colonization or infection with MRSA, as well as differences in the clinical or demographic characteristics and antimicrobial susceptibility observed for community- or hospital-origin infection. Soft tissue samples were used for bacteriological culture in all cases, even though a bone biopsy may be a better sample in patients with osteomyelitis (Senneville et al., 2006). However, surgical excision can be potentially traumatic for the patient, and samples taken from the base of the wound after debridement are adequate for identifying the infecting organism (Kessler et al., 2006; Pellizzer et al., 2001; Perry et al., 1991). Accordingly, we adopted the current procedure.

Over the last 3 years, the number of isolates in our foot clinic has agreed with that found in a previous report (Dang et al., 2003), averaging 1.4 isolates per case, which is still lower than that found in other studies (Goldstein et al., 1996; Lipsky et al., 1990). The present study confirms the findings of previous studies in which Gram-positive aerobes were predominant in diabetic foot infections (Dang et al., 2003; Mantey et al., 2000). S. aureus was the most common pathogen among the Gram-positive bacteria isolated from ulcers (Tentolouris et al., 2006), and almost two-thirds $(63.4 \%)$ of $S$. aureus isolates were MRSA. However, the ratios of Gram-positive aerobes to Gramnegative aerobes and MRSA to $S$. aureus, and the prevalence of $S$. aureus, differed from the above-mentioned reports. These dissimilarities could be attributed to the differences in age/gender composition, ulcer grades and study settings between our study patients and those of previous studies.

This study demonstrated that a high proportion of HAMRSA patients $(66.7 \%)$ were identified among the diagnosed MRSA patients in our institution. Although

Table 4. Antimicrobial susceptibility patterns of MRSA isolates from infected foot ulcers in diabetic patients $(n=26)$

All strains of MRSA were uniformly susceptible to the study drugs.

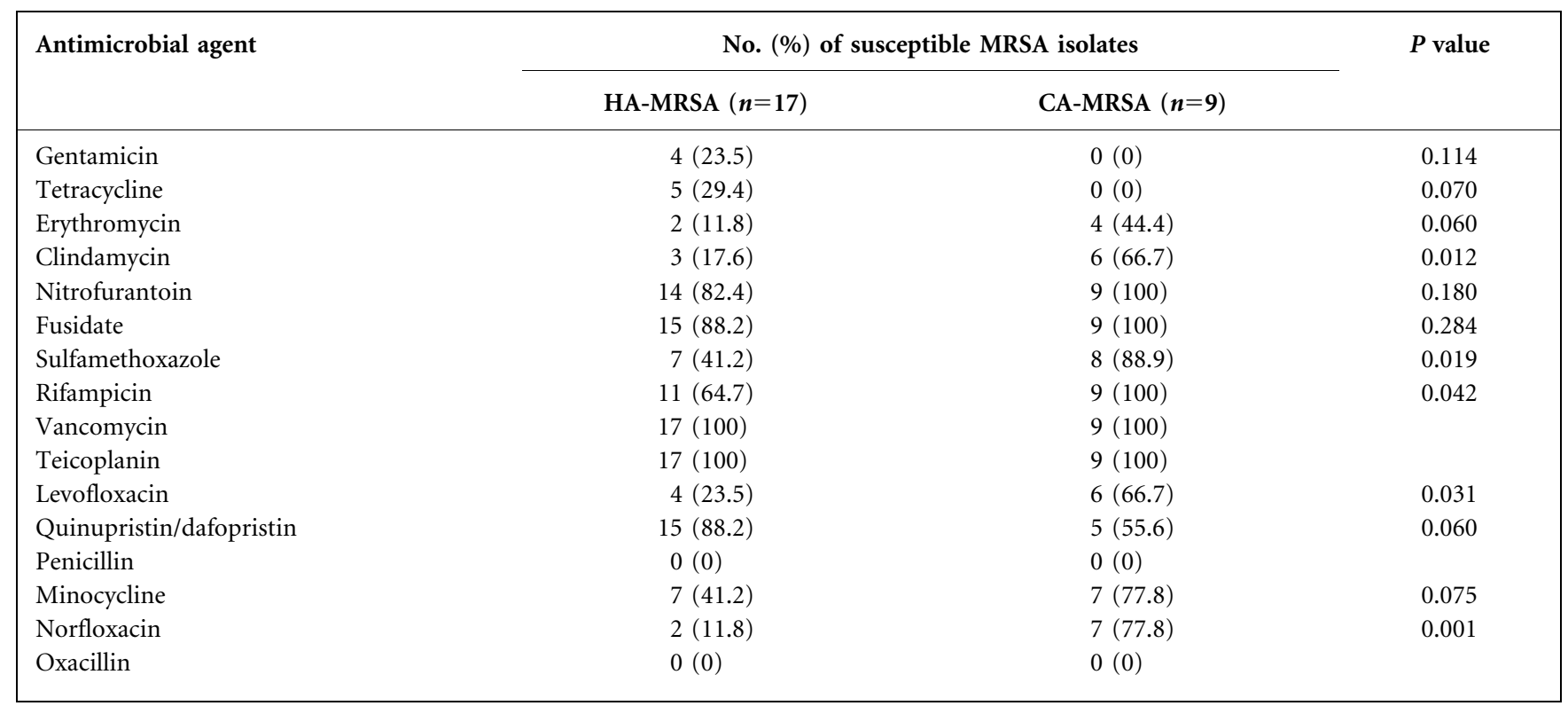


previous studies have indicated that there were no differences in ulcer size between ulcers positive for meticillin-sensitive $S$. aureus and MRSA (Tentolouris et al., 1999), in the current study, ulcer size was an independent predictor of MRSA infection. Furthermore, osteomyelitis was another predictive factor of MRSA infection. This finding is consistent with previous studies in which $S$. aureus (Embil et al., 2006; Senneville et al., 2006, 2008), including MRSA (AragónSánchez et al., 2009), was the most commonly found bacterium causing foot osteomyelitis in diabetic patients. Furthermore, $65.4 \%$ of all MRSA isolates were identified as HA-MRSA in our institution. CA-MRSA was also recognized in diabetic foot ulcers, accounting for about $6 \%$ of the patients. CA-MRSA was reported to account for some $12 \%$ of the cases identified in a study from the USA (Naimi et al., 2003). There is a suggestion that CA-MRSA may be more virulent (Baba et al., 2002). However, there is currently little evidence to support the view that the involvement of CAMRSA affects the outcome of diabetic foot ulcers. Patients with CA-MRSA infections often do not exhibit risk factors known in patients with HA-MRSA infections. These risk factors include recent hospitalization, dialysis, nursing-home residence and other co-morbid conditions, such as diabetes (Fridkin et al., 2005; Naimi et al., 2003). In the current study, however, none of the CA-MRSA or HA-MRSA patients were on renal replacement therapy, and none had resided in a chronic-care facility within the previous 3 months. Our data showed that no significant effect could be found for these factors (including diabetes), which have been shown previously to predispose patients towards colonization with HA-MRSA in the diabetic foot. The factors predisposing patients toward HA-MRSA infection included age, duration of diabetes and glucose control.

Our data also indicated that infection with HA-MRSA resulted in a prolonged hospital stay. An increased hospital stay may be due to the higher prevalence of diabetic complications, including neuropathy, peripheral vascular disease and osteomyelitis, in patients infected with HAMRSA; surgery was always required in these patients. This is reinforced by the fact that HA-MRSA isolates were more resistant to multiple antibiotics, such as clindamycin, sulfamethoxazole, rifampicin, levofloxacin and norfloxacin, compared with CA-MRSA isolates. We noticed a slight difference between CA-MRSA and HA-MRSA in susceptibility to multiple antibiotics such as erythromycin, fusidate, nitrofurantoin and minocycline, which were more active against CA-MRSA. Moreover, although HA-MRSA displayed more sensitivity to quinupristin/dafopristin, the difference did not reach statistical significance. Consistent with other investigations, we found vancomycin and teicoplanin to be highly active, whilst penicillin was inactive against MRSA, with no differences in susceptibility observed for isolates of community or hospital origin. These findings will be helpful in assessing the appropriate empirical antibiotic regimen for diabetic foot ulcers infected with MRSA in order to shorten hospital stays and reduce costs.
There are several limitations to our study. First, this was a healthcare-based retrospective case series study. Thus, we were unable to estimate the true prevalence and antimicrobial susceptibility profiles of CA-MRSA and HAMRSA infections in general diabetic foot ulcers of the Chinese population. Secondly, although medical charts were carefully reviewed, the absence of personal interviews made it possible to misclassify MRSA acquisition due to lack of a detailed history of hospital-related exposures and medical treatment, especially for those patients whose charts were incomplete.

In conclusion, we believe that this is the first Chinese study to highlight CA- and HA-MRSA infections in diabetic foot ulcers. The prevalence of MRSA was alarmingly high, especially for HA-MRSA. The size of the ulcer and osteomyelitis were independent predictors of MRSA infection, and HA-MRSA infection reflected increased diabetic complications. The age of patients, duration of diabetes and glucose control appeared to be factors that predisposed patients toward HA-MRSA. HA-MRSA was associated with an increased requirement for surgical treatment. These findings suggest that prospective multicentre studies are required to assess the appropriate empirical antibiotic regimen for diabetic foot ulcers, taking into consideration the results found here for HA- and CAMRSA infections in diabetic foot ulcers in a Chinese care hospital. In addition, the results alert us to the need for proper management of antibiotics to decrease the incidence of MRSA infection in this population, to reduce the risk of running out of effective antimicrobials for patients.

\section{ACKNOWLEDGEMENTS}

We would like to thank the nursing staff of the Division of Endocrinology, Zhongda Hospital of Southeast University, for their help. This work was supported partially by the National Nature Science Foundation of China (no. 30870870 to S.-H.W. and no. 30600206 to Y.-J. G.) and the Nature Science Foundation of JiangSu Province (no. BK2008302).

\section{REFERENCES}

Aragón-Sánchez, J., Lázaro-Martínez, J. L., Quintana-Marrero, Y., Hernández-Herrero, M. J., Garcia-Morales, E., Cabrera-Galván, J. J. \& Beneit-Montesinos, J. V. (2009). Are diabetic foot ulcers complicated by MRSA osteomyelitis associated with worse prognosis? Outcomes of a surgical series. Diabet Med 26, 552-555.

Baba, T., Takeuchi, F., Kuroda, M., Yuzawa, H., Aoki, K., Oguchi, A., Nagai, Y., Iwama, N., Asano, K. \& other authors (2002). Genome and virulence determinants of high virulence community-acquired MRSA. Lancet 359, 1819-1827.

Dang, C. N., Prasad, Y. D., Boulton, A. J. \& Jude, E. B. (2003). Methicillin-resistant Staphylococcus aureus in the diabetic foot clinic: a worsening problem. Diabet Med 20, 159-161.

Embil, J. M., Rose, G., Trepman, E., Math, M. C., Duerksen, F., Simonsen, J. N. \& Nicolle, L. E. (2006). Oral antimicrobial therapy for diabetic foot osteomyelitis. Foot Ankle Int 27, 771-779. 
Fridkin, S. K., Hageman, J. C., Morrison, M., Sanza, L. T., ComoSabetti, K., Jernigan, J. A., Harriman, K., Harrison, L. H., Lynfield, R. \& other authors (2005). Methicillin-resistant Staphylococcus aureus disease in three communities. N Engl J Med 352, 1436-1444.

Game, F. \& Jeffcoate, W. (2004). MRSA and osteomyelitis of the foot in diabetes. Diabet Med 21, 16-19.

Goldstein, E. J., Citron, D. M. \& Nesbit, C. A. (1996). Diabetic foot infections. Bacteriology and activity of $10 \mathrm{oral}$ antimicrobial agents against bacteria isolated from consecutive cases. Diabetes Care 19, 638-641.

Hartemann-Heurtier, A., Robert, J., Jacqueminet, S., Ha, V. G., Golmard, J. L., Jarlier, V. \& Grimaldi, A. (2004). Diabetic foot ulcer and multidrug-resistant organisms: risk factors and impact. Diabet Med 21, 710-715.

Kessler, L., Piemont, Y., Ortega, F., Lesens, O., Boeri, C., Averous, C., Meyer, R., Hansmann, Y., Christmann, D. \& other authors (2006). Comparison of microbiological results of needle puncture vs. superficial swab in infected diabetic foot ulcer with osteomyelitis. Diabet Med 23, 99-102.

Lipsky, B. A., Pecoraro, R. E., Larson, S. A., Hanley, M. E. \& Ahroni, J. H. (1990). Outpatient management of uncomplicated lower-extremity infections in diabetic patients. Arch Intern Med 150, 790-797.

Lipsky, B. A., Berendt, A. R., Embil, J. \& De Lalla, F. (2004). Diagnosing and treating diabetic foot infections. Diabetes Metab Res Rev 20, S56-S64.

Mantey, I., Hill, R. L., Foster, A. V., Wilson, S., Wade, J. J. \& Edmonds, M. E. (2000). Infection of foot ulcers with Staphylococcus aureus associated with increased mortality in diabetic patients. Commun Dis Public Health 3, 288-290.

Naimi, T. S., LeDell, K. H., Como-Sabetti, K., Borchardt, S. M., Boxrud, D. J., Etienne, J., Johnson, S. K., Vandenesch, F., Fridkin, S. \& other authors (2003). Comparison of community- and health careassociated methicillin-resistant Staphylococcus aureus infection. JAMA 290, 2976-2984.
NCCLS (2002). Performance Standards for Antimicrobial Susceptibility Testing, 12th Informational Supplement. M100-S12. Wayne, PA: National Committee for Clinical Laboratory Standards.

Pellizzer, G., Strazzabosco, M., Presi, S., Furlan, F., Lora, L., Benedetti, P., Bonato, M., Erle, G. \& de Lalla, F. (2001). Deep tissue biopsy vs. superficial swab culture monitoring in the microbiological assessment of limb-threatening diabetic foot infection. Diabet Med 18, 822-827.

Perry, C. R., Pearson, R. L. \& Miller, G. A. (1991). Accuracy of cultures of material from swabbing of the superficial aspect of the wound and needle biopsy in the preoperative assessment of osteomyelitis. J Bone Joint Surg Am 73, 745-749.

Senneville, E., Melliez, H., Beltrand, E., Legout, L., Valette, M., Cazaubiel, M., Cordonnier, M., Caillaux, M., Yazdanpanah, Y. \& Mouton, Y. (2006). Culture of percutaneous bone biopsy specimens for diagnosis of diabetic foot osteomyelitis: concordance with ulcer swab cultures. Clin Infect Dis 42, 57-62.

Senneville, E., Lombart, A., Beltrand, E., Valette, M., Legout, L., Cazaubiel, M., Yazdanpanah, Y. \& Fontaine, P. (2008). Outcome of diabetic foot osteomyelitis treated nonsurgically: a retrospective cohort study. Diabetes Care 31, 637-642.

Shankar, E. M., Mohan, V., Premalatha, G., Srinivasan, R. S. \& Usha, A. R. (2005). Bacterial etiology of diabetic foot infections in South India. Eur J Intern Med 16, 567-570.

Tentolouris, N., Jude, E. B., Smirnof, I., Knowles, E. A. \& Boulton, A. J. (1999). Methicillin-resistant Staphylococcus aureus: an increasing problem in a diabetic foot clinic. Diabet Med 16, 767-771.

Tentolouris, N., Petrikkos, G., Vallianou, N., Zachos, C., Daikos, G. L., Tsapogas, P., Markou, G. \& Katsilambros, N. (2006). Prevalence of methicillin-resistant Staphylococcus aureus in infected and uninfected diabetic foot ulcers. Clin Microbiol Infect 12, 186-189.

Wagner, F. W., Jr (1981). The dysvascular foot: a system for diagnosis and treatment. Foot Ankle 2, 64-122. 